\title{
Phytochemicals derived from soya bean husk exert hypoglycemic and anti-adipogenic properties in cell culture models
}

\begin{abstract}
Purpose: Literature has shown that phenolic acids and flavonoids are bearing with hypoglycemic and anti-adipogenic properties. Therefore, this study aims to evaluate the possibility of phenolic-rich soya bean husk powder extract (SHPE) in combating diabetes and obesity using in vitro models.

Design/methodology/approach: The hypoglycemic properties were evaluated by determining the ability of SHPE $(25-100 \mu \mathrm{g} / \mathrm{mL})$ in inhibiting a-amylase and a-glucosidase enzymes and in triggering insulin secretion in BRIN-BD1 1 cells. Murine 3T3-L1 adipocytes were used for evaluating the anti-adipogenic properties of SHPE through the determination of relative lipid accumulation, triglyceride content and glycerol-3-phosphate dehydrogenase (GPDH) activity.

Findings: The hypoglycemic properties of SHPE was in the dose-dependent manner, where $100 \mu \mathrm{g}$ SHPE/mL exhibited a significant higher $(\mathrm{p}<0.05)$ a-amylase inhibitory activity $(56.8$ \pm 0.11 per cent $)$ and insulin secretion activity $(0.73 \pm 0.02 \mu \mathrm{g} / \mathrm{l})$ against other concentrations. In contrast to the aforementioned findings, a significant lower a-glucosidase inhibitory activity (52.0 \pm 0.44 per cent) was also observed in $100 \mu \mathrm{g}$ SHPE/mL. Nevertheless, findings revealed that all the SHPE were able to inhibit the activity of a-amylase and a-glucosidase and stimulated the insulin secretion in BRIN-BD11 cells. On the other hand, the antiadipogenic properties of SHPE were in the reverse dose-dependent manner, where $100 \mu \mathrm{g}$ $\mathrm{SHPE} / \mathrm{mL}$ demonstrated a significant lower $(\mathrm{p}<0.05)$ relative lipid accumulation $(48.5 \pm$ 0.03 per cent), intracellular triglyceride content $(5.7 \pm 0.07 \mathrm{mg} / \mathrm{dL})$ and GPDH activity $(1.0 \pm$ $0.01 \mathrm{mU} / \mathrm{mL}$ ). These findings reflected that $100 \mu \mathrm{g} \mathrm{SHPE} / \mathrm{mL}$ was a potent anti-adipogenic agent when compared with other concentrations. In conclusion, soya husk could emerge as a potential hypoglycemic and anti-adipogenic agents in in vitro models.

Originality/value: This was the first study to explore the effectiveness of phytochemicals derived from soya bean husk in ameliorating hyperglycemia and adipogenesis. Promising findings that derived from the present study could enable the scientists to re-evaluate the potential use of agricultural wastes, especially in the formulation of nutraceuticals.
\end{abstract}

Keyword: Adipogenesis; BRIN BD-11; Hypoglycemic; 3T3-L1 adipocytes; Soya bean husk 